\title{
Participatory Disaster Recovery Simulation Modeling for Community Resilience Planning
}

\author{
Scott B. Miles ${ }^{1}$
}

Published online: 10 December 2018

(C) The Author(s) 2018

\begin{abstract}
A major challenge in enhancing the resilience of communities stems from current approaches used to identify needs and strategies that build the capacity of jurisdictions to mitigate loss and improve recovery. A new generation of resilience-based planning processes has emerged in the last several years that integrate goals of community well-being and identity into recovery-based performance measurement frameworks. Specific tools and refined guidance are needed to facilitate evidence-based development of recovery estimates. This article presents the participatory modeling process, a planning system designed to develop recovery-based resilience measurement frameworks for community resilience planning initiatives. Stakeholder engagement is infused throughout the participatory modeling process by integrating disaster recovery simulation modeling into community resilience planning. Within the process, participants get a unique opportunity to work together to deliberate on community concerns through facilitated participatory modeling. The participatory modeling platform combines the DESaster recovery simulation model and visual analytics interfaces. DESaster is an open source Python Library for creating discrete event simulations of disaster recovery. The simulation model was developed using a human-centered design approach whose goal is to be open, modular, and extensible. The process presented in this article is the first participatory modeling approach for analyzing recovery to aid creation of community resilience measurement frameworks.
\end{abstract}

Scott B. Miles

milessb@uw.edu

1 Department of Human Centered Design and Engineering, University of Washington, Seattle, WA 98195, USA
Keywords Community resilience planning $\cdot$ Disasters $\cdot$ Disaster recovery $\cdot$ Participatory modeling $\cdot$ Recovery-based performance targets $\cdot$ Simulation modeling

\section{Introduction}

A growing number of jurisdictions are conducting resilience planning initiatives that engage a broad range of stakeholders to identify needs and strategies to mitigate impacts from extreme hazard events and improve recovery from disasters. Agencies and organizations have begun to synthesize the lessons from these initiatives and publish recommended resilience planning and policy analysis procedures. For example, the U.S. National Institute of Standards and Technology (NIST) Community Resilience Planning Guide lays out how to approach a community resilience planning process (NIST 2016). The NIST guide was written based on the premise that measurement of community resilience requires collaboratively quantifying hazard losses, social impacts, recovery objectives, and the influence of resilience planning strategies. The core of the NIST guide is the creation of a community resilience measurement framework, which requires estimates of expected and target recovery times for different community and infrastructure sectors. To better implement the NIST guide, new tools and refined guidance are needed to facilitate analytically-based development of recovery estimates and subsequent resilience performance measurement frameworks.

This article presents a conceptual framework for identifying and evaluating community resilience strategies through a participatory process that implements the NIST 
guide, which incorporates a human-centered approach to disaster recovery modeling. The framework's intent is to have community representatives and external stakeholders directly involved in the design, use, and evaluation of recovery simulation modeling in order to envision plausible disaster recovery trajectories related to future hazard events. It incorporates a participatory modeling approach for analyzing recovery to aid the deliberation of community resilience measurement frameworks.

In the following section, the core component of the NIST resilience planning process-development of a recovery-based performance measurement framework-is synthesized. A brief overview of participatory modeling is provided in the third section. The fourth section of the article analyzes the state-of-the-art of disaster recovery modeling to consider how modeling can be integrated in a community resilience planning process that generally follows the NIST guide. The new recovery simulation model DESaster is detailed in the fifth and sixth sections. Prior to conclusion, the seventh section details a high-level process agenda for utilizing a participatory modeling approach to develop community resilience measurement frameworks.

\section{Community Resilience Planning and Recovery- Based Performance Targets}

The NIST Community Resilience Planning Guide (NIST 2016) was motivated by three jurisdictional-level seismic resilience planning initiatives: San Francisco Bay Area Planning and Urban Research Association's Resilient City (SPUR) initiative (Poland 2009), Resilient Washington State-RWS-(WASSC 2012), and ORP, the Oregon Resilience Plan (OSSPAC 2013). SPUR was a city-scale initiative, while RWS and ORP were state-scale initiatives.

The NIST guide defines community resilience as "the ability of a community to prepare for anticipated hazards, adapt to changing conditions, withstand, and recover rapidly from disruptions" (NIST 2016, p. 9). The SPUR, RWS, and ORP adopted their own unique but similar definitions of resilience. Ultimately, all four definitions are based on the "resilience triangle" conception of Bruneau et al. (2003) — the capacity to reduce loss and aid recovery.

Innovated by SPUR, the most prominent and groundbreaking feature of the NIST guide, as well as RWS and ORP, is development of community performance measurements frameworks. These take the form of timetables showing expected and target post-event recovery times for key community sectors. While SPUR applied this framework primarily to mitigation of the built environment (buildings and lifeline infrastructure), each subsequent initiative extended the approach to consider other aspects of community functioning.
The SPUR, RWS, and ORP initiatives developed their own set of criteria to define "recovery" for each different sector. The NIST guide similarly suggests that communities develop their own criteria to define recovery for each considered sector. For example, RWS defined recovery for the "critical services" sector as "time it ought to take to restore the component to $80-90 \%$ operational" (WASCC 2012 , p. 17). Alternatively, three levels of recovery criteria were defined for the transportation sector: (1) minimal ("[...] minimum level of service is restored, primarily for the use of emergency responders, repair crews, and vehicles transporting food and other critical supplies"); (2) functional ("Service [...] is sufficient to get the economy moving again"); and (3) operational ("Restoration is up to 80-90\% of capacity: A full level of service has been restored and is sufficient to allow people to commute to school and to work."). The SPUR and ORP initiatives took a similarly custom and sector-specific approach to define and operationalize recovery.

Miles (2018b) found that the time, effort, participation, analysis, and systematicness used for each resilience planning initiative to determine the recovery estimates for the respective resilience measurement frameworks varied considerably. For the SPUR initiative, expected and target recovery times were informally estimated by a small number of experts on their mitigation task force based on their knowledge, experience, and outside consultation. The SPUR initiative did not follow a systematic analytical process for estimating expected and target recovery times. Over 50 participants were involved in the RWS process, but with an equally ad hoc and experiential approach. Neither recovery data from past disaster nor analytical models were used in the RWS initiative to inform recovery estimates. The ORP process included almost twice as many participants. Each ORP task group was free to determine their own approach for estimating recovery times. Some ORP task groups utilized the United States Federal Emergency Management Agency's Hazus loss estimation software (FEMA 2017), as well as recovery data from past disasters. This was the only initiative to explicitly use quantitative data or analysis (for example, modeling).

Miles (2018b) reorganized each initiative's measurement frameworks and converted recovery time estimates into common units (days) to facilitate comparison. Estimates for both current and target recovery times differ widely across the initiatives in most instances. It is difficult to reconcile inconsistencies given both differences and similarities in jurisdictional scales, level of mitigation activity, and hazard exposure. Miles (2018b) concluded that a more systematic, analytically-based approach to developing the performance measurement framework is needed. 
The NIST guide advances the prior initiatives' approaches by suggesting that jurisdictions develop their resilience measurement frameworks with additional consideration of community-defined goals and the needs of the community's social institutions. The guide does not prescribe an explicit analytic scheme to integrate with their stakeholder deliberation process to link resilience objectives to individual projects or detailed strategies. Similar to the SPUR, RWS, and ORP initiatives, the NIST guide does not advise on how to make resilience performance measurement frameworks operational-that is, measure progress of whether implemented recommendations are closing gaps between estimated current and target recovery times. The variability in recovery time estimation outcomes from the SPUR, RWS, and ORP initiatives, as well as the noted shortcomings of the NIST guide, warrant research and development of processes that better integrate systematic analysis and stakeholder participation to formulate improved recovery-based resilience measurement frameworks.

\section{Participatory Modeling}

Models of hazards and disasters are being increasingly used in planning processes, such as FEMA's Hazus being used to inform the Oregon Resilience Plan initiative. In general, these processes have been used to help develop disaster preparedness, emergency response, and hazard mitigation policies and plans - rather than pre- or post-event recovery plans (Miles et al. 2019). To best realize the benefits of the approach laid out in the NIST guide, the approach should be conceptualized, researched, and applied to incorporate participatory modeling.

Participatory modeling integrates computer modeling, natural science, social science, and stakeholder participation-most commonly researched and applied in the content of environmental management (Gray et al. 2017). Computational models used in participatory processes, rather than just scientific inquiry or engineering design, should help to formulate and measure goals effectively, as well as evaluate strategies to reach these goals (Norton 1996). For participatory modeling to appropriately inform environmental policy and planning, Norton (1996 p. 125) argues that

[m]odels [should] improve our understanding in the specific sense that they illuminate environmental problems and allow us to focus on those natural dynamics that are causally related to important social values. $[. .$.$] The [\ldots]$ models chosen $[\ldots]$ must $[. .$. help us to understand nature, but they must also encourage us to understand nature in a way [...that assists us] to formulate and measure [...] goals effectively and to propose and implement policies to achieve these goals.

Participatory modeling provides meaningful opportunities for involvement and input from local stakeholders who typically do not have computer modeling expertise and are not directly consulted. It shifts the focus of both experts and stakeholders from scientific or engineering proof to a contextualized deliberation and inquiry (Fischer 2000). Fostering participation in modeling promotes an iterative process and living products. Broad participation can legitimize the development and implementation of a model or any decision, plan, or policy based on a model (Korfmacher 2001). Computer models intended for use within participatory processes related to policy or planning should be designed to be transparent and open (Miles 2000, 2011).

Miles (2011) adopted and evaluated the seven transparency components of Drew (2003) as criteria for the design of computer models intended to support participatory policy and planning processes. Drew (2003) originally developed the components to better understand the use of geographic information systems within complex participatory decision-making processes. Applying these criteria to participatory modeling, computer models should be designed to be: (1) clear; (2) integrated; (3) accessible; (4) truthful and accurate; (5) logical and rational; (6) accountable; and (7) open. Arias et al. (2000) defines openness in the context of modeling as affording opportunities to stakeholders to make reasonable changes to the modeling system or process (interfaces, visualizations, software, algorithms, and data). As such, models cannot be completely implemented prior to use in a participatory modeling processes because modelers cannot anticipate every need without a human-centered design process. Stakeholders should be frequently involved in the modeling (Korfmacher 2001), but it is critical that their roles are clear (Ehrman and Stinson 1999). Stakeholders do not need modeling and expertise can directly inform model application requirements (for example, what aspects or geographic extents to model), data sources, parameter constraints, scenario logics (for example, what variable or parameter values to manipulate and how), and expectations for model evaluation, among other modeling choices. While models can and should evolve from stakeholder input, not all aspects of a model can or should be subject to stakeholder direction. 


\section{Disaster Recovery Modeling for Community Resilience Planning}

The relative infrequency of catastrophic hazard events and the complexity of community recovery from disasters make quantitative data on long-term recovery trends relatively scarce. Thus, it is difficult to consult case study recovery data to inform creation of performance measurement frameworks as advocated in the NIST guide. Readily available modeling tools, such as FEMA's Hazus, provide inadequate representation of recovery from earthquakes (Miles and Chang 2006; Miles and Chang 2011). Considering this, application and advancements of recoveryspecific models are necessary for a more rigorous approach to implementing the deliberative planning process laid out in the NIST guide.

The WISC framework provides one of the most comprehensive outlines of potential sectors or topics of disaster recovery (Miles 2015). The framework is called WISC after four of its eight constructs: well-being, identity, services, and capitals. The remaining constructs are metabolism (the flow of resources), geography, sufficiency (supply-demand dynamics), and complexity. The WISC constructs are used below to synthesize the state-of-the-art of disaster recovery modeling.

Very few, if any, disaster recovery models exist that represent variables of community well-being or public health. Some existing approaches to disaster recovery modeling, such as regression-based models, are not conducive to representing changes in identity or adaptation (for example, reaching a "new normal"). More recent simulation approaches, however, can facilitate understanding changes in the identity of communities or systems (Nejat and Damnjanovic 2012; Davis 2013; Grinberger and Felsenstein 2014; Huling and Miles 2015; Eid and ElAdaway 2017). A small number of models exist that were developed to represent service or sufficiency variables, such as serviceability, quality of supply (not just quantity), demand, and satisfaction (Davis 2013; Miles 2014; Nojima and Kato 2014). The large majority of recovery models represent geography to some degree, but few are topologically explicit (Nejat and Damnjanovic 2012; Ouyang and Zhao 2014). Metabolism is a focus of many lifeline restoration models (Chang et al. 2002; Çağnan et al. 2006), but is less emphasized in recovery models of other sectors.

A community's capital—for example, built, economic, social, and human-is most commonly used to distinguish categories of recovery models. Of all potential types of capital, built capital is most frequently modeled. Modeling of lifeline infrastructure has been researched most often compared to all other recovery topics. The sectors of these recovery models include power systems (Çağnan and
Davidson 2007), water systems (Davis 2013), and transportation networks (Lee and Kim 2007). Recovery models of built capital have been developed to represent the reconstruction of residential building stocks (Miles and Chang 2006, 2011; Nejat and Damnjanovic 2012; Grinberger and Felsenstein 2014; Huling and Miles 2015; Nejat and Ghosh 2016; Eid and El-Adaway 2017) and, less commonly, commercial building stocks (Miles and Chang 2006, 2011; Hwang et al. 2016).

Comparably fewer recovery models related to economic, social, human, and political capital have been researched, but cover a broader range of sectors. Most of these models are narrowly-scoped dynamic economic impact models (Santos et al. 2014), but also relate to issues of business cycling (Hallegatte and Ghil 2008), workforce disruption (Haimar and Santos 2015), reconstruction investment (Xie et al. 2014), population dynamics (Grinberger and Felsenstein 2014), policy outcomes (Yasui et al. 2014), and humanitarian relief (Gonçalves 2011). It appears that only one disaster recovery model explicitly represents the influence of post-event natural capital dynamics (Frazier et al. 2013). There are several models that relate multiple types of community capital (Miles and Chang 2006, 2011; Frazier et al. 2013; Grinberger and Felsenstein 2014; Santos et al. 2014).

\section{Human-Centered Disaster Recovery Simulation Model}

The recovery modeling community is progressively adopting simulation-based approaches (Miles et al. 2019). Simulation models are well suited to post-event recovery modeling because empirical data is not a necessity for their development, unlike, for example, regression models. Simulation models can leverage conceptual or qualitative insights of disaster recovery and so compensate for a scarcity of empirical data needed to train machine-learning models. Simulation models are typically more interpretable and focused on the understanding of flows, behaviors, and processes, rather than prediction.

Discrete event simulation (DES) is a modeling approach that is useful for representing macro-level recovery that spans relatively-long timeframes and includes multiple individual recovery processes that have well-defined temporal character and community capital (resource) dependencies (Miles et al. 2019). Representing recovery as a set of distinct temporal processes with quantified duration that are explicitly tied to well-defined quantifiable resources lends itself to participatory modeling. This is because of the potential for users to select, assemble, and parameterize these distinct model elements (conceptually and programmatically) into a context-specific simulation. 
Huling and Miles (2015) describe a proof-of-concept discrete event simulation modeling approach for exploring post-earthquake housing recovery scenarios. Subsequent work led to the first version of the open source recovery simulation library DESaster ${ }^{1}$ (Miles 2018a). Currently, the deployable codebase of DESaster exclusively represents housing recovery related to single-family wood-frame houses; modules for simulating utility infrastructure recovery are under development (Ganji and Miles 2018). DESaster is implemented in the Python programming language and has been designed to be transparent, modular, and extensible. For example, different types of model elements can be reviewed, chosen, and combined in custom ways to represent generalizable aspects of disaster recovery. DESaster is built on top of the open-source discrete event simulation Python Library called SimPy. ${ }^{2}$

SimPy and DESaster use a process-based approach to DES, which is illustrated in Fig. 1. SimPy requires specification of the simulation environment (boundary constraints), modeled entities (for example, owners), entity attributes (for example, owner savings), entity states (for example, displacement from house), processes (for example, building inspection), events (for example, inspection approval), and resources (for example, buildings, loan processors). Three generalized types of resources can be represented in SimPy: (1) resources that can be used by a limited number of entities or processes at a time (for example, a building inspector); (2) resources related to the production and consumption of a homogeneous, undifferentiated stock (for example, money); and (3) resources that allow the production and consumption of other entities or processes (for example, complex resources such as a "housing stock"). Process interactions between entities and resources are conceptualized as discrete events. The state of one or more process (for example, loan application) results in the triggering or interruption of one or more process (for example, home repair), as well as the modification of one or more resource (for example, a household's savings account).

Represented entities in DESaster currently include owner occupiers, renters, and landlords. Other recovery stakeholders, such as FEMA or insurance companies, are implicitly represented as resource management of human and financial resources. Financial resources can be private, shared, competitive, or "infinite" and includes entities' savings, FEMA individual assistance, U.S. Small Business Administration (SBA) loans, and insurance policies. Human resources in DESaster currently includes building inspectors, SBA loan processors, FEMA individual assistance processors, insurance adjusters, permit application

\footnotetext{
${ }^{1}$ https://github.com/milessb/DESaster.

${ }^{2}$ https://simpy.readthedocs.io/.
}

processors, engineers, and contractors. Discrete events for entities largely consist of making requests for human or financial resources (or both) and having those requests fulfilled to repair or rebuild their residence. Other events include beginning to search for a different residence (temporary, transitional, or permanent) if the entity's residence is uninhabitable, and successfully occupying that new residence.

DESaster is a modular library for building simulations and so does not impose a hard-coded progression toward recovery, which is well-suited to NIST's recommendation that communities developed their own sector-specific definitions of recovery. The different types of entities, resources, requests, and events can be arranged in different ways by the user. For example, one simulation can be built that requires an owner to submit an insurance claim prior to applying for FEMA individual assistance, while a different simulation built with DESaster can allow owners to make both requests simultaneously (and then, for example, reimburse FEMA, if necessary). DESaster has operations that can represent decision logic for entities. For example, an entity can be imbued with patience related to each resource request or search for residence. If the duration of an event exceeds that patience tolerance (for example, 90 days of patience to secure a local housing rental), the current event is interrupted and a new event triggered (for example, search outside the jurisdiction for a rental).

Example output from a DESaster simulation is shown in Fig. 2, which illustrates the outputs for a single entity, in this case an owner named Selena, whose data and parameters are simulated for illustration purposes only. The figure shows the times that the owner put in a request for different types of human and financial capital and received the results of their request (if it was successfully fulfilled). DESaster can monitor and record all state changes and event triggers within a simulation run. As a result, a user can choose to produce a narrative story for individual entities (quoted text in Figs. 2 and 3), which creates a compelling means of interpreting simulation outputs.

Data is required for DESaster to quantify model parameters, resource characteristics, and entity attributes. Key parameters are durations associated with represented recovery processes. Some currently required durations are the length of time needed to mobilize inspectors, process insurance claims, process assistance requests, process loan applications, process a building permit request, and rebuild a wood-frame house, among others. Other parameters include the potential states that public and private entities and capital resources can take on. Resource characteristics relate to the quantity and quality of modeled resourcesfor example, the number of building inspectors and characteristics of the housing stock, including Hazus-generated building-damage states. Probability distributions are used 


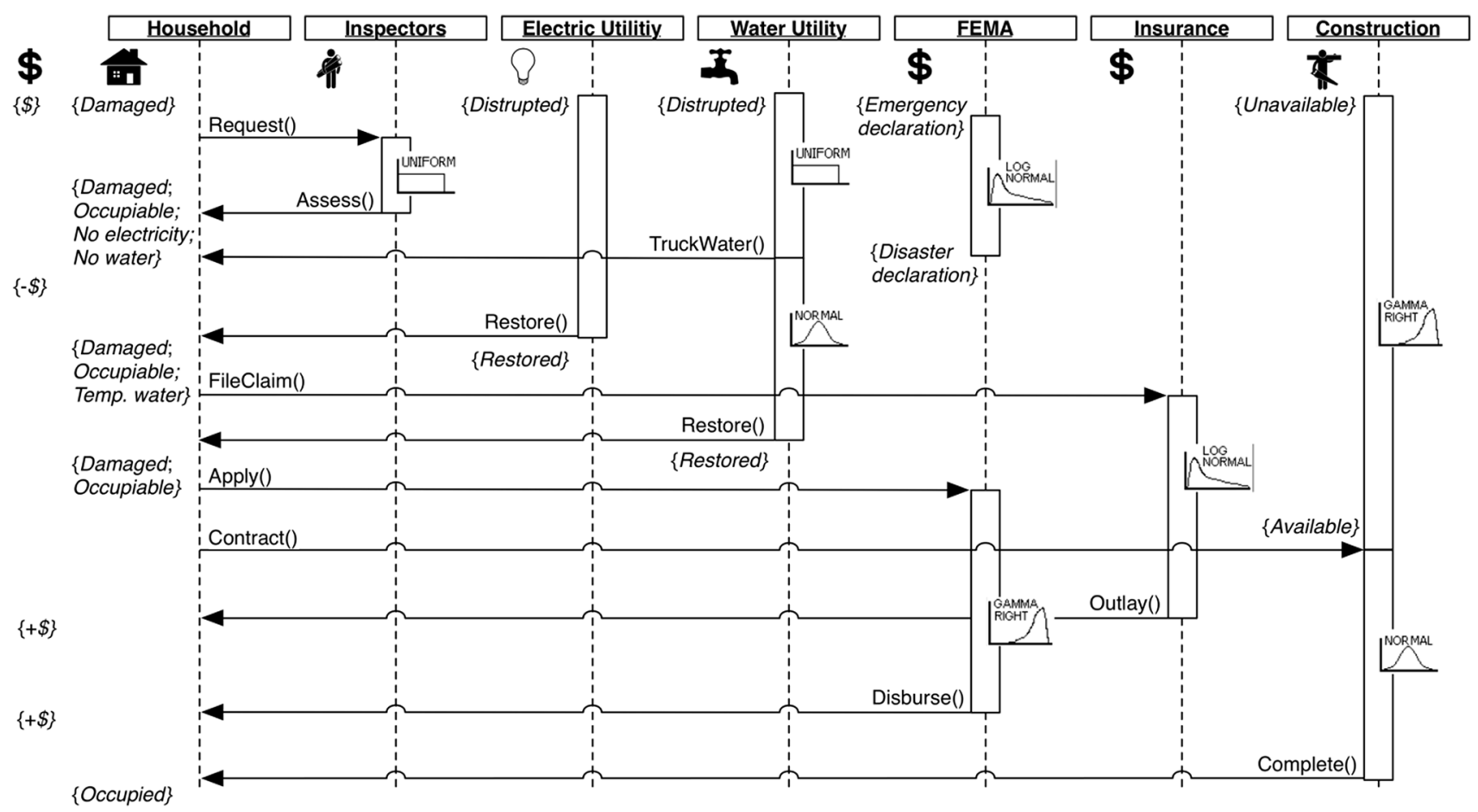

Fig. 1 Illustration of a process-based discrete event simulation of recovery. The vertical dimension represents increasing time in the downward direction. Rectangles represent processes with a probabilistic duration associated with one or more discrete events. Icons represent shared resources, with states indicated in curly brackets \{\} . This illustration does not include all possible recovery entities, resources, and processes for housing or household recovery
Fig. 2 Entity-scale output from a DESaster simulation showing times that the entity either put in a request (_put) or got the requested resource (_get). For this example, the owner's name is Selena; her story reflects the plotted discrete event recovery events and was automatically generated by DESaster

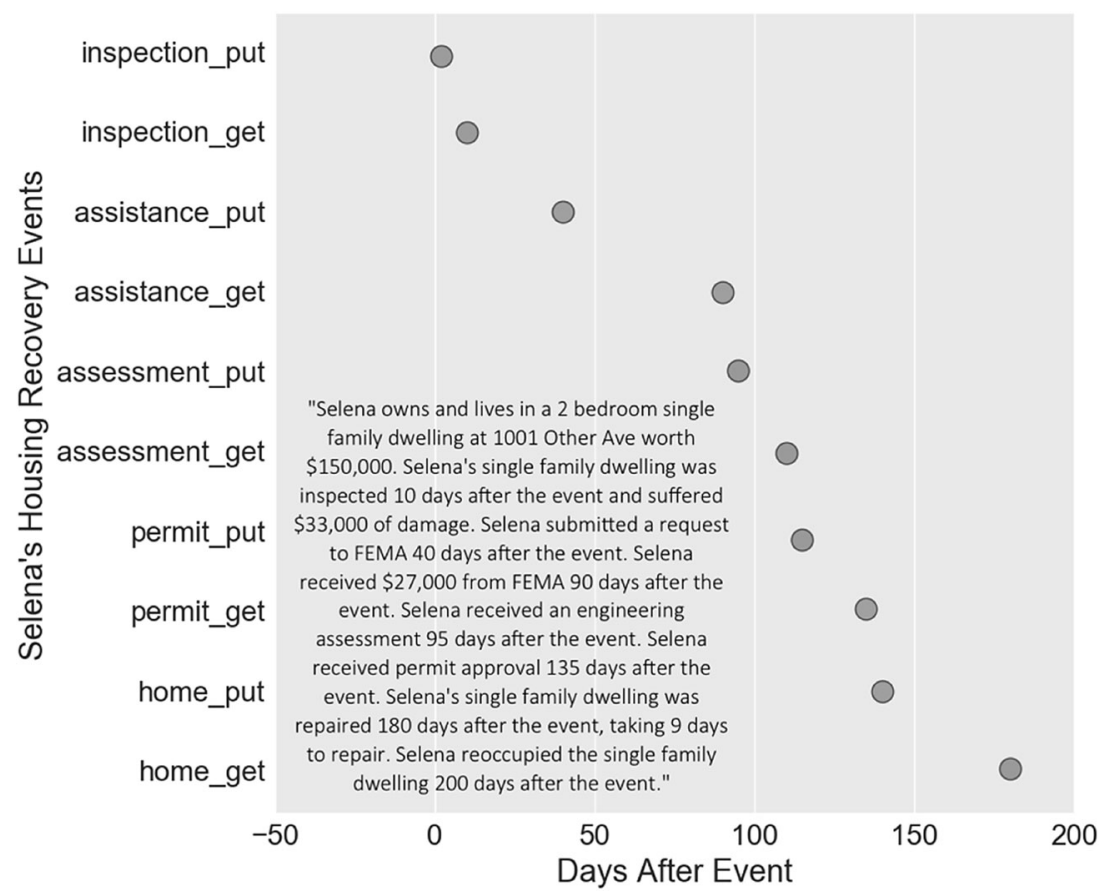

For many of the durations and resource characteristics, representative quantitative and qualitative data can be readily obtained from relevant organizations (for example, typical building permit processing times for a jurisdiction). 


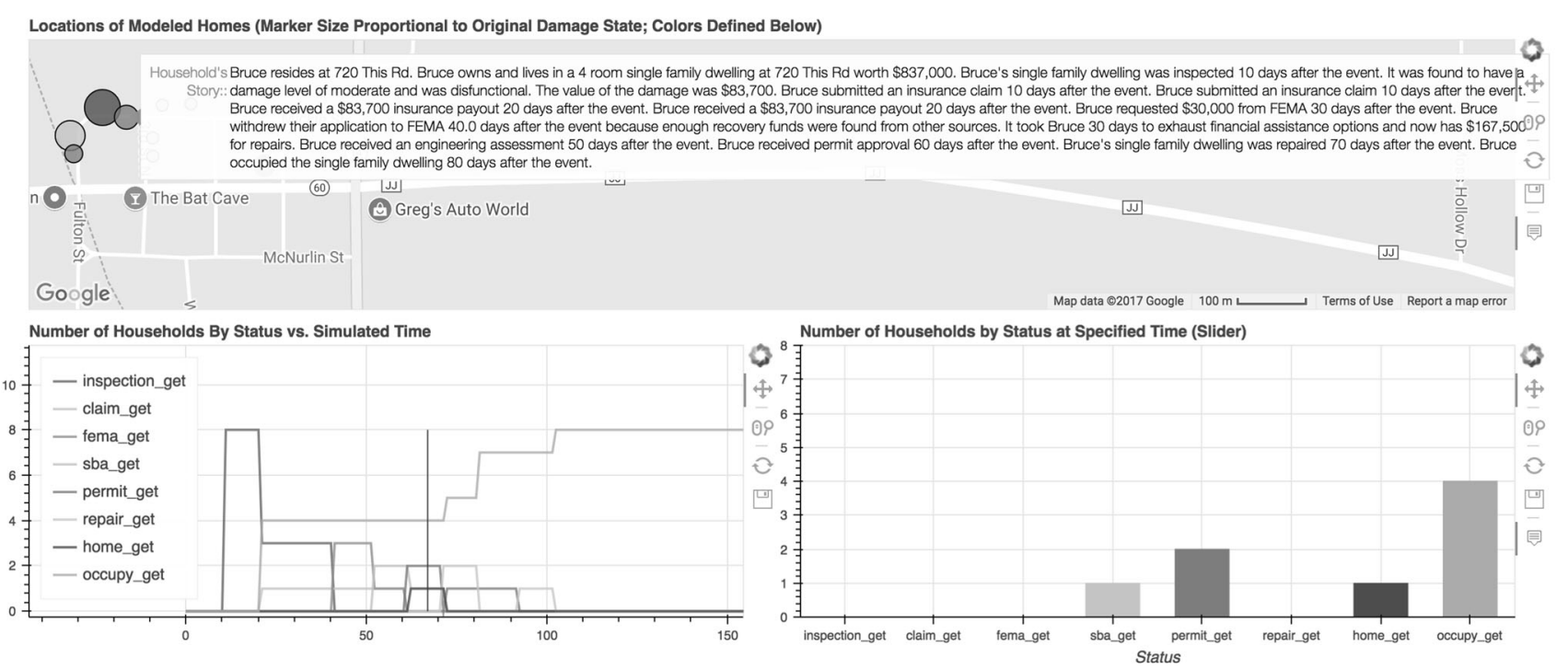

DAY: 67

Fig. 3 Alpha prototype of a component of a browser-based visual analytics dashboard for DESaster as part of a participatory modeling platform

An appealing aspect of the design of DESaster is the convenience in which model parameters, entity attributes, and resource characteristics can be quantified using expert elicitation methods. There are a range of well-tested methods for eliciting probabilistic data from experts (Cooke and Goossens 2004; Goossens et al. 2008) and software to facilitate use of these methods (Morris et al. 2014).

\section{Participatory Modeling Platform and Human- Centered Design}

Representation of community resilience that is conceptualized as disaster loss and recovery is hyperdimensional. It involves potentially several tens of community neighborhoods, thousands or millions of households and businesses - each with a range of attributes - and many recovery indicators that vary over multiple temporal and spatial scales (Miles 2014). Within a simulation modeling context, this hyperdimensionality explodes with the creation of alternative "what if" scenarios, for example to determine a community's expected and target recovery capacities as part of a resilience planning process informed by the NIST guide. For usability, this hyperdimensionality must be packaged and presented in a transparent and open way (Arias et al. 2000; Drew 2003).

To make DESaster suitable for participatory modeling, interactive and visual interfaces are needed that are created using human-centered design principles and implemented to be flexible, adaptable, and extensible (Ganji and Miles 2018). Little to no formal research has been done to develop visual analytics that facilitate understanding of community resilience relationships and dependencies between hazard loss and disaster recovery data (simulated or empirical) or to understand the implications of scenariobased processes across different spatiotemporal scales (Miles 2014).

Figure 3 shows a preliminary browser-based visual analytics interface for exploring simulation outputs that is included with the DESaster Python Library (Miles 2018a). The interface is implemented using the Python Library Bokeh. The design was informed by user research interviews of 10 Seattle-area emergency managers, as part of an ongoing, iterative, human-centered design process. The interviews were conducted by six University of Washington undergraduate students for a university course on data visualization. The interview protocol centered on the following open-ended questions to better understand the problem space:

(1) How do data visualizations help you in your workflow?

(2) How do/would you visualize indicators during a disaster?

(3) How do/would you use these data visualizations to monitor and make decisions?

(4) What indicators do/would you monitor to understand recovery?

The interview transcripts were compiled, but not systematically analyzed, to inform low-fidelity mockups (sketches) of alternative interface designs, as well as to provide a reference for implementing the prototype. 
Further design iterations of interface prototypes for visualizing DESaster outputs are ongoing (Ganji and Miles 2018).

While DESaster is a Python code library, typical users would assemble, parameterize, and run particular simulations using Jupyter. ${ }^{3}$ Jupyter is an open-source interactive programming environment, which is well suited to be a platform for participatory modeling. Jupyter is interfaced using "notebooks," which are collections of individual text cells where users can enter executable code written in multiple different programming languages (for example, Python, R, or Julia), rich text, formatted mathematical equations, interactive plots, animations, web links, and most other common web content. Jupyter was developed to promote reproducible science (Ustyuzhanin et al. 2017), match the exploratory workflow of data science (Kluyver et al. 2016), and provide a computational platform for academic and public education (Hamrick 2016).

To support participatory recovery modeling using DESaster, Jupyter notebooks can be extended and served via JupyterHub, similar to Evans et al. (2016) who created an interactive neuroscience simulation modeling platform using Jupyter and JupyterHub. With JupyterHub, multiple instances of the same Jupyter notebook can be served from a central host or cloud computing service (Fernández and Andersson 2016). With JupyterHub, users do not have to install any modeling environments or programming libraries to do computation and visualization because all dependencies are installed on the host server. Multiple users can interact with the same notebook but make their own temporary or persistent modifications that do not change the original hosted notebook. As an example, Hamrick (2016) extended the Jupyter notebook format and combined it with JupyterHub to create Nbgrader (notebook grader). Nbgrader allows instructors to: (1) create an assignment notebook; (2) host the notebook on a personal server or cloud service; (3) have users spawn their own notebook and complete their assignment; (4) view each user's completed notebook; (5) evaluate and comment on each individual cell; and (6) allow users to view their evaluated notebooks.

Within a resilience planning workshop setting, JupyterHub will allow small groups of participants to separately construct and interact with a DESaster simulation via their own instances of a Jupyter notebook in similar manner as Nbgrader. As described in the following section, this would be done as part of exercises that encourage participants to explore simulated recovery times (for example, for housing reconstruction) for different scenarios (for example, a different number of building inspectors or different recovery funding policies). With notebooks submitted by each

\footnotetext{
${ }^{3}$ http://jupyter.org/.
}

participant, it is straightforward with JupyterHub for process facilitators to view and combine each participant's submissions (constructed simulations, input parameter values, and simulation outputs) to inform the group's creation of a collective resilience performance measurement framework. Description is given below about how participatory modeling using DESaster, Jupyter, and JupyterHub can be situated within a facilitated resilience planning process.

\section{Participatory Modeling Process for Community Resilience Planning}

A participatory modeling process for use in community resilience planning is proposed in this section. The participatory modeling process is designed to assist participants in creating a recovery-based resilience measurement framework like the ones developed for the SPUR, RWS, and ORP resilience planning initiatives and advocated in the NIST Community Resilience Planning Guide for Buildings and Infrastructure Systems. The process assists participants to reveal key interdependencies and tradeoffs that may hamper or promote their community's functioning and future recovery.

The proposed process was synthesized with insights from the three resilience planning initiatives and the NIST guide in a manner that can best incorporate the presented participatory modeling platform. The process is comprised of multiple advisory group calls and participant workshops. An advisory group is formed to help define the process invitation list, advocate for local participation, indicate potential data sources, provide feedback on the process guide design, help interpret workshop outcomes, give feedback to synthesize outcomes, approve final community deliverables, and provide general advice. Examples of process participants include representatives from local chambers of commerce, community-based organizations, local elected officials, government agency managers, utilities organizations, and major regional employers.

Under the guidance of the advisory group, the process facilitators gather as much relevant data as possible prior to the first process workshop. Most critical public data include census data, tax assessor data, public utilities data, time series from past disasters (for example, electricity restoration durations), and damage/loss estimates. Initially, DESaster is parameterized using these data, as well as simulated data (for example, simulate household-level data from census block data). Process participants finalize default parameter distributions for process durations (for example, duration distribution for wood-frame house construction time) and resource magnitudes (for example, number of contractors to build a wood-frame house). This 
can be done by facilitating participants through a process to elicit parameter estimates or constraints-commonly referred to as expert elicitation. Process participants also finalize choices for data sources and input data, particularly to define resilience strategy scenarios to simulate and compare.

The DESaster Library serves as the basis for participatory modeling for the resilience planning process. As part of the analytic-deliberative process, the DESaster Library is adapted and expanded based on participant feedback and workshop discussions of community concerns. This is facilitated by DESaster's modular design and reorganizes a core aspect of participatory modeling-user-defined models. But participation is also a higher risk element of the process compared to past community-resilience planning initiatives. Implementation of user-defined features is constrained by feasibility factors, such as time and knowledge limitations.

The proposed participatory modeling process is outlined below as six half-day workshops, with a brief agenda given for each workshop. The workshops would be conducted over six to, more likely, twelve months.

Workshop 1. (1) Introductions, (2) recovery and resilience discussion, (3) discussion about impacted communities, (4) identify additional participants, (5) scope process;

Workshop 2. (1) Review Hazus scenarios, (2) DESaster demonstration, (3) discuss DESaster features, (4) identify data sources, (5) introduce post-workshop online parameter elicitation assignment;

Workshop 3. (1) DESaster training, (2) review elicitation results, (3) participatory DESaster simulations for current/expected recovery capacities, (4) introduce post-workshop online parameter elicitation assignment;

Workshop 4. (1) Review final DESaster results for current recovery capacities, (2) review elicitation results, (3) participatory DESaster simulations for target/desired recovery capacities, (4) identify community needs and resilience strategies, (5) introduce post-workshop online parameter elicitation assignment;

Workshop 5. (1) Review final DESaster results for target recovery capacities, (2) review elicitation results, (3) participatory DESaster simulations to evaluate resilience strategies evaluation;

Workshop 6. (1) Review final DESaster results for resilience strategies, (2) discuss and/or prioritize modeled resilience strategies,
(3) identify additional strategies (that is, that couldn't be modeled), (4) discuss deliverable requirements and next steps.

The facilitator role is critical to the overall process; such facilitators will need to be familiar with the NIST Community Resilience Planning Guide, have a working understanding of DESaster, be proficient with Jupyter, and be capable of effectively facilitating groups. As noted in Siebenhüner and Barth (2005), this may require two or more people - a process facilitator and a model moderator. Between workshops, process facilitators synthesize workshop notes, compile additional data, develop additional DESaster features, set up and facilitate any online expert elicitation assignments, conduct final recovery simulations, finalize workshop agendas, create workshop presentations, and convene advisory group calls to solicit continuous feedback during the process. The final process and workshop agendas would be agreed on by the advisory group and process facilitators, with potential input from participants.

\section{Conclusion}

This article presents a more systematic and analyticallybased approach to development of recovery-based resilience measurement frameworks in comparison to past community resilience planning initiatives and the NIST's Community Resilience Planning Guide. Collaboration between researchers, community representatives, and external stakeholders is a requirement of NIST's advocated approach. Collaboration and stakeholder engagement are infused throughout the presented participatory modeling process for integrating disaster recovery simulation into community resilience planning. As part of the presented process, participants get a unique opportunity to work together to deliberate on community concerns through facilitated modeling.

The participatory modeling process presented here is supported by a platform built on the recovery simulation modeling library of DESaster (Miles 2018a). The modeling platform combines DESaster, a new visual analytics interface, and a cloud-based computation environment called JupyterHub. The process involves experts and stakeholders in simulation modeling, scenario development, and evaluation of interventions to mitigate against and recover from future disasters. As part of future resilience planning processes, additional features will be developed for DESaster as part of the participatory modeling process. That is, DESaster will be further improved and expanded in direct response to input and expressed needs of planning participants. The intention is that 
improvements done during future resilience planning processes are integrated into the open source codebase of DESaster. This will serve to provide continuous evaluation and development of DESaster, while expanding its feature set and applicability.

Acknowledgements Funding support for this article was provided by National Science Foundation Awards \#1560939 and \#1541025.

Open Access This article is distributed under the terms of the Creative Commons Attribution 4.0 International License (http://crea tivecommons.org/licenses/by/4.0/), which permits unrestricted use, distribution, and reproduction in any medium, provided you give appropriate credit to the original author(s) and the source, provide a link to the Creative Commons license, and indicate if changes were made.

\section{References}

Arias, E., H. Eden, G. Fischer, A. Gorman, and E. Scharff. 2000. Transcending the individual human mind-creating shared understanding through collaborative design. ACM Transactions on Computer-Human Interaction 7(1): 84-113.

Bruneau, M., S.E. Chang, R.T. Eguchi, G.C. Lee, T.D. O'Rourke, A.M. Reinhorn, M. Shinozuka, K. Tierney, W.A. Wallace, and D. von Winterfeldt. 2003. A framework to quantitatively assess and enhance the seismic resilience of communities. Earthquake Spectra 19(4): 733-752.

Chang, S.E., W.D. Svekla, and M. Shinozuka. 2002. Linking infrastructure and urban economy: Simulation of water-disruption impacts in earthquakes. Environment and Planning B: Planning and Design 29(2): 281-301.

Cooke, R.M., and L.H.J. Goossens. 2004. Expert judgement elicitation for risk assessments of critical infrastructures. Journal of Risk Research 7(6): 643-656.

Çağnan, Z., and R.A. Davidson. 2007. Discrete event simulation of the post-earthquake restoration process for electric power systems. International Journal of Risk Assessment and Management 7(8): 1138-1156.

Çağnan, Z., R.A. Davidson, and S.D. Guikema. 2006. Post-earthquake restoration planning for Los Angeles Electric Power. Earthquake Spectra 22(3): 589-608.

Davis, C.A. 2013. Quantifying post-earthquake water system functionality. In Proceedings of the sixth China-Japan-US trilateral symposium on lifeline earthquake engineering, ed. C. Davis, X. Du, M. Miyajima, and L. Yan, 19-26. Reston, VA: American Society of Civil Engineers.

Drew, C.H. 2003. Transparency - considerations for PPGIS research and development. URISA Journal 15(1): 73-78.

Ehrman and Stinson. 1999. Joint fact-finding and the use of technical experts. In The consensus building handbook, ed. L. Susskind, S. McKearnan, and J. Thomas-Larmer. Thousand Oaks, CA: Sage.

Eid, M.S., and I.H. El-Adaway. 2017. Sustainable disaster recovery: Multiagent-based model for integrating environmental vulnerability into decision-making processes of the associated stakeholders. Journal of Urban Planning and Development 143(1): Article 04016022

Evans, B.D., S. Jarvis, S.R. Schultz, and K. Nikolic. 2016. PyRhO: A multiscale optogenetics simulation platform. Frontiers in Neuroinformatics $10(1)$ : Article 8 .

FEMA (Federal Emergency Management Agency). 2017. Hazus: FEMA's methodology for estimating potential losses from disasters. https://www.fema.gov/hazus. Accessed 29 Nov 2017.
Fernández, L., and R. Andersson. 2016. Jupyterhub at the ESS: An interactive Python computing environment for scientists and engineers. Proceedings of the seventh international particle accelerator conference, 8-13 May 2016, Busan, Korea.

Fischer, F. 2000. Citizens, experts, and the environment. Durham, NC: Duke University Press.

Frazier, A.E., C.S. Renschler, and S.B. Miles. 2013. Evaluating postdisaster ecosystem resilience using MODIS GPP data. International Journal of Applied Earth Observation and Geoinformation 21: 43-52.

Ganji, A., and S.B. Miles. 2018. Human-centered simulation modeling for critical infrastructure disaster recovery planning. Proceedings of the Global Humanitarian Technology Conference, 18-21 October 2018, San Jose, CA, USA.

Gray, S., M. Paolisso, R. Jordan, and S. Gray. 2017. Environmental modelling with stakeholders: Theory, methods, and applications. New York: Springer.

Goossens, L.H.J., R.M. Cooke, A.R. Hale, and L. Rodić-Wiersma. 2008. Fifteen years of expert judgement at TUDelft. Safety Science 46(2): 234-244.

Gonçalves, P. 2011. Balancing provision of relief and recovery with capacity building in humanitarian operations. Operations Management Research 4(1-2): 39-50.

Grinberger, A.Y., and D. Felsenstein. 2014. Bouncing back or bouncing forward? Simulating urban resilience. Urban Design and Planning 167(DP3): 115-124.

Haimar, E.A., and J.R. Santos. 2015. A stochastic recovery model of influenza pandemic effects on interdependent workforce systems. Natural Hazards 77(2): 987-1011.

Hallegatte, S., and M. Ghil. 2008. Natural disasters impacting a macroeconomic model with endogenous dynamics. Ecological Economics 68(1-2): 582-592.

Hamrick, J.B. 2016. Creating and grading IPython/Jupyter notebook assignments with NbGrader. In Proceedings of the the 47th ACM technical symposium, ed. C. Alphonce, and J. Tims, 242. New York: ACM Press.

Huling, D., and S.B. Miles. 2015. Simulating disaster recovery as discrete event processes using python. In Proceedings of the 2015 IEEE global humanitarian technology conference (GHTC), 9-12 October 2015, Seattle, WA, USA.

Hwang, S., M. Park, H.S. Lee, and S.H. Lee. 2016. Hybrid simulation framework for immediate facility restoration planning after a catastrophic disaster. Journal of Construction Engineering and Management 142(8): Article 04016026.

Kluyver, T., B. Ragan-Kelley, and F. Perez. 2016. Jupyter notebooks-a publishing format for reproducible computational workflows. In Positioning and power in academic publishing players, agents and agendas, ed. F. Loizides, and B. Schmidt, 87-90. Amsterdam, The Netherlands: IOS Press.

Korfmacher, K.S. 2001. The politics of participation in watershed modeling. Environmental Management 27(2): 161-176.

Lee, J.S., and T.J. Kim. 2007. Implementation of spatiotemporal model for infrastructure reconstruction strategy under large-scale disaster. Transportation Research Record: Journal of the Transportation Research Board 2022: 39-46.

Miles, S.B. 2000. Towards policy relevant environmental modeling: Contextual validity and pragmatic models. United States Geological Survey open-file report 00-401. Reston, VA: U.S. Department of the Interior.

Miles, S.B. 2011. Participatory model assessment of earthquakeinduced landslide hazard models. Natural Hazards 56(3): 749-766.

Miles, S.B. 2014. Modeling and visualizing infrastructure-centric community disaster resilience. Proceedings of the 10th U.S. national conference on earthquake engineering: Frontiers of 
earthquake engineering, 21-25 July 2014, Anchorage, AK, USA.

Miles, S.B. 2015. Foundations of community disaster resilience: Well-being, identity, services, and capitals. Environmental Hazards 14(2): 103-121.

Miles, S.B. 2018a. A Python library for discrete event simulation of disaster recovery (version v0.1.1-alpha). Zenodo. http://doi.org/ 10.5281/zenodo.1190513. Accessed 3 Dec 2018.

Miles, S.B. 2018b. Comparison of jurisdictional seismic resilience planning initiatives. PLOS Currents Disasters. https://doi.org/10. 1371/currents.dis.42c24f29588cb4f887af021449949801.

Miles, S.B., and S.E. Chang. 2006. Modeling community recovery from earthquakes. Earthquake Spectra 22(2): 439-458.

Miles, S.B., and S.E. Chang. 2011. ResilUS: A community based disaster resilience model. Cartography and Geographic Information Science 38(1): 36-51.

Miles, S.B., H.V. Burton, and H. Kang. 2019. Community of practice for modeling disaster recovery. Natural Hazards Review 20(1): Article 04018023.

Morris, D.E., J.E. Oakley, and J.A. Crowe. 2014. A web-based tool for eliciting probability distributions from experts. Environmental Modelling and Software 52: 1-4.

Nejat, A., and I. Damnjanovic. 2012. Agent-based modeling of behavioral housing recovery following disasters. ComputerAided Civil and Infrastructure Engineering 27(10): 748-763.

Nejat, A., and S. Ghosh. 2016. LASSO model of postdisaster housing recovery: Case study of Hurricane Sandy. Natural Hazards Review 17(3): Article 04016007.

NIST (National Institute of Standards and Technology). 2016. Community resilience planning guide for buildings and infrastructure systems. NIST Special Publication 1190. Volume I. Washington, DC: U.S. Department of Commerce. https:// nvlpubs.nist.gov/nistpubs/SpecialPublications/NIST.SP.1190v1. pdf. Accessed 3 Dec 2018.

Nojima, N., and H. Kato. 2014. Modification and validation of an assessment model of post-earthquake lifeline serviceability based on the Great East Japan Earthquake Disaster. Journal of Disaster Research 9(2): 108-120.
Norton, B.G. 1996. Integration or reduction. In Environmental pragmatism, ed. A. Light, and E. Katz, 105-138. Abingdon, UK: Routledge.

OSSPAC (Oregon Seismic Safety Policy Advisory Commission). 2013. The Oregon Resilience Plan. Salem, OR: Oregon Seismic Safety Policy Advisory Committee.

Ouyang, M., and L. Zhao. 2014. Do topological models contribute to decision making on post-disaster electric power system restoration? Chaos: An Interdisciplinary Journal of Nonlinear Science 24(4): Article 043131.

Poland, C. 2009. The resilient city: Defining what San Francisco needs from its seismic mitigation policies. San Francisco, CA: San Francisco Planning \& Urban Research Association.

Santos, J.R., K.D.S. Yu, S.A.T. Pagsuyoin, and R.R. Tan. 2014. Time-varying disaster recovery model for interdependent economic systems using hybrid input-output and event tree analysis. Economic Systems Research 26(1): 60-80.

Siebenhüner, B., and V. Barth. 2005. The role of computer modelling in participatory integrated assessments. Environmental Impact Assessment Review 25(4): 367-389.

Ustyuzhanin, A., T.D. Head, I. Babuschkin, and A. Tiunov. 2017. Everware toolkit. Supporting reproducible science and challenge-driven education, arXiv.org, 1703.01200.

WASSC. 2012. Resilient Washington State: A framework for minimizing loss and improving statewide recovery after an earthquake. Olympia, WA: State of Washington Emergency Management Council Seismic Safety Committee. http://mil.wa. gov/other-links/seismic-safety-committee-ssc. Accessed 3 Dec 2018.

Xie, W., N. Li, J.D. Wu, and X.L. Hao. 2014. Modeling the economic costs of disasters and recovery: analysis using a dynamic computable general equilibrium model. Natural Hazards and Earth System Science 14(4): 757-772.

Yasui, T., S. Shirasaka, and T. Maeno. 2014. Designing critical policy infrastructures by participatory systems analysis: The case of Fukushima's reconstruction. International Journal of Critical Infrastructures 10(3-4): 334-336. 